\title{
The International Temperature Scale of 1948
}

\author{
By H. F. Stimson
}

\begin{abstract}
The International Temperature Scale is based upon six fixed and reproducible equilibrium temperatures to which numerical values have been assigned and upon specified interpolation formulas relating temperature to the indications of specified measuring instruments. This is the first revision of the scale adopted in 1927. It is designed to conform as nearly as practicable to the thermodynamic centigrade scale as now known, while incorporating certain refinements based on experience to make the scale more uniform and reproducible than its predecessor. A new value for the temperature of the silver point and the use of Planck's law with a new value for the radiation constant, $c_{2}$, are the only changes which produce significant effects on numerical values assigned to temperatures.
\end{abstract}

The first International Temperature Scale was adopted by the Seventh General Conference on Weights and Measures in 1927, and is now known as the International Temperature Scale of $1927 .{ }^{1}$ The General Conference on Weights and Measures is the diplomatic body, representing 33 participating nations, which has power to adopt recommendations concerning standards of weights and measures for international use. The recommendations are made by the International Committee on Weights and Measures which nominally consists of 18 scientists (not more than one from any nation) elected by the General Conference. The General Conference is scheduled to meet at intervals of 6 years and the International Committee at intervals of 2 years.

The Seventh Genéral Conference recommended that international thermometric conferences be called by the International Committee to revise the temperature scale as occasion required. The Eighth General Conference, in 1933, made official provision for these conferences. In June 1937 the International Committee approved the creation of an Advisory Committee on Thermometry, subject to the sanction of the Ninth General Conference.

A meeting of the Advisory Committee was first

\footnotetext{
${ }^{1}$ Comptes Rendus des Séances de la Septième Conférence Générale des
Poids et Mesures, p. 94 (1927); also in Travaux et Mémoires du Bureau inter-

1 Comptes Rendus des Séances de la Septième Conférence Générale des
Poids et Mesures, p. 94 (1927); also in Travaux et Mémoires du Bureau international des Poids et Mesures 18 (1930); also George K. Burgess, E S J. Research 1, 635 (1928) RP22.
}

called in July 1939. It approved a revision of the text of the scale of 1927 for proposal to the International Committee, but no action was taken because World War II began before the date originally scheduled for the Ninth General Conference. At the call of the International Committee, the Advisory Committee met again in May 1948. At this meeting three resolutions and a new text for the International Temperature Scale were approved.

Both the International Committee and the Ninth General Conference met in October 1948. On the recommendation of the International Committee, the General Conference sanctioned the creation of this Advisory Committee, which is to be known as the "Advisory Committee on Thermometry and Calorimetry". The General Conference then examined and adopted the resolutions which had been proposed by the Advisory Committee and had been transmitted by the International Committee with minor modifications.

The first of these resolutions concerns the International Temperature Scale directly and is as follows:

"1. With the present-day technique, the triple point of water is susceptible of being a more precise thermometric reference point than the 'melting point of ice'.

"The Advisory Committee considers, there- 
fore, that the zero of the thermodynamic centigrade scale should be defined as being the temperature 0.0100 degree below that of the triple point of pure water."

The Conference also adopted the International Temperature Scale of 1948 as proposed by the Advisory Committee on Thermometry and Calorimetry; and, on this occasion, decided to give to the degree of temperature the designation of degree Celsius in place of degree centigrade or centesimal. Before the end of 1948 the official text of the scale was approved for publication in the Procès-Verbaux des Séances du Comité international des Poids et Mesures, t. XXI, 1948.

The National Bureau of Standards, therefore, in common with other national laboratories, began using the definitions of the International Temperature Scale of 1948 on January 1, 1949, both in its own scientific work and for calibrating instruments for other scientific and industrial purposes. It is recommended that scientists and industrial workers elsewhere conform to the definitions of the revised scale as set forth in the text.

A translation of the official text follows.

\section{Text of The International Temperature Scale of 1948}

\section{Part I. Introduction}

The Kelvin scale, on which temperatures are designated as ${ }^{\circ} \mathrm{K}$ and denoted by the symbol $T$, is recognized as the fundamental thermodynamic scale to which all temperature measurements should ultimately be referable. On this scale the interval from the ice point, $T_{0}$, to the steam point, $T_{100}$, is 100 degrees. The Kelvin scale and the thermodynamic centigrade ${ }^{2}$ scale, on which the temperature is $T-T_{0}$, are hereby adopted, in principle, by the Ninth General Conference on Weights and Measures. Any temperature interval expressed on one of these scales will have the same numerical value as when expressed on the other.

The experimental difficulties inherent in the measurement of temperature on the thermodynamic scale led to the adoption in 1927, by the Seventh General Conference on Weights and Measures, of a practical scale which was designated as the

\footnotetext{
2 The General Conference, held in October 1948, decided to discontinue the use of the words "centesimal" and "centigrade" and to replace them by "Celsius".
}

International Temperature Scale. This scale was intended to be as nearly identical with the - thermodynamic centigrade scale as was possible with the knowledge then available. It was designed to be conveniently and accurately reproducible and to provide means for specifying any temperature on the International Scale within much narrower limits than was possible on the thermodynamic scale.

The scale herein. defined represents the first revision of the scale adopted in 1927 and is designed to conform as nearly as practicable to the thermodynamic centigrade scale as now known, while incorporating certain refinements, based on experience, to make the scale more uniform and reproducible than its predecessor.

The experimental procedures by which the scale is to be realized are substantially unchanged. Only two of the revisions in the definition of the scale result in appreciable changes in the numerical values assigned to measured temperatures. The change in the value for the silver point from $960.5^{\circ}$ to $960.8^{\circ} \mathrm{C}$ changes temperatures measured with the standard thermocouple. The adoption of a different value for the radiation constant, $c_{2}$, changes all temperatures above the gold point, while the use of the Planck radiation formula instead of the Wien formula affects the very high temperatures. The Planck formula is consistent with the thermodynamic scale and consequently removes the upper limit which was imposed by Wien's law in the 1927 scale.

Other important modifications, which cause little or no change in the numerical values of temperatures, but serve to make the scale more definite and reproducible are (a) the termination of one part of the scale at the oxygen point instead of at $-190^{\circ} \mathrm{C}$, (b) the division of the scale at the freezing point of antimony (about $630^{\circ} \mathrm{C}$ ) instead of at $660^{\circ} \mathrm{C}$, (c) the requirements for higher purity of the platinum of the standard resistance thermometer and standard thermocouple, and for smaller permissible limits for the electromotive force of the standard thermocouple at the gold point.

The scale defined by the resistance thermometer remains substantially identical with the 1927 scale. In the range between $630^{\circ}$ and $1063^{\circ} \mathrm{C}$, numerical values of temperature on the 1948 scale are higher than on the 1927 scale, the maximum difference being about 0.4 degree near $800^{\circ} \mathrm{C}$. 
Temperatures on the International Temperature Scale of 1927 were designated as ${ }^{\circ} \mathrm{C}$ or ${ }^{\circ} \mathrm{C}$ (Int.). Inasmuch as the designation "C" is retained in this revision, it should be applied in the future to temperatures on the last scale adopted previous to the time at which the designation is used. Where any doubt might arise, the designation should be specified as ${ }^{\circ} \mathrm{C}$ (Int. 1927) or ${ }^{\circ} \mathrm{C}$ (Int. 1948).

\section{Part II. Definition of The International Temperature Scale of 1948}

1. Temperatures on the International Temperature Scale of 1948 will be designated as "O C" or "० C (Int. 1948)" and denoted by the symbol, $t$.

2. The scale is based upon a number of fixed and reproducible equilibrium temperatures (fixed points) to which numerical values are assigned, and upon specified formulas for the relations between temperature and the indications of the instruments calibrated at these fixed poin.ts.

3. The fixed points and the numerical values assigned to them are given in Table I. These values, in each case, define the equilibrium temperature corresponding to a pressure of 1 standard atmosphere, defined as 1,013,250 dynes $/ \mathrm{cm}^{2}$. The last decimal place given for each of the values of the primary fixed points only represents the degree of reproducibility of that fixed point.

TABLE I.-Fundamental and primary fixed points under the standard pressure of 1,013,250 dynes $/ \mathrm{cm}^{2}$

Temperature ${ }^{\circ} \mathrm{C}$

(a) Temperature of equilibrium between liquid oxygen and its vapor (oxygen point) _ _ _ -182.970

(b) Temperature of equilibrium between ice and air saturated water (ice point) (Fundamental fixed point)

(c) Temperature of equilibrium between liquid water and its vapor (steam point) (Fundamental fixed point)

(d) Temperature of equilibrium between liquid sulfur and its vapor (sulfur point) _.....

(e) Temperature of equilibrium between solid and liquid silver (silver point) _._._._. 960.8

(f) Temperature of equilibrium between solid and liquid gold (gold point)

1063. 0

4. The means available for interpolation lead to a division of the scale into four parts.

(a) From $0^{\circ} \mathrm{C}$ to the freezing point of antimony the temperature, $t$, is defined by the formula

$$
R_{t}=R_{0}\left(1+A t+B t^{2}\right)
$$

where $R_{t}$ is the resistance, at temperature, $t$, of the platinum resistor between the branch points formed by the junctions of the current and potential leads of a standard resistance thermometer. The constant, $R_{0}$, is the resistance at $0^{\circ} \mathrm{C}$, and the constants, $A$ and $B$, are to be determined from measured values of $R_{t}$ at the steam and sulfur points. The platinum in a standard resistance thermometer shall be annealed, and of such purity that $R_{100} / R_{0}$ is greater than 1.3910 .

(b) From the oxygen point to $0^{\circ} \mathrm{C}$, the temperature, $t$, is defined by the formula

$$
R_{t}=R_{0}\left[1+A t+B t^{2}+C(t-100) t^{3}\right],
$$

where $R_{t}, R_{0}, A$, and $B$ are determined in the same manner as in (a) above, and the constant, $C$, is calculated from the measured value of $R_{t}$ at the oxygen point.

(c) From the freezing point of antimony to the gold point, the temperature, $t$, is defined by the formula

$$
E=a+b t+c t^{2},
$$

where $E$ is the electromotive force of a standard thermocouple of platinum and platinum-rhodium alloy when one junction is at $0^{\circ} \mathrm{C}$ and the other is at the temperature, $t$. The constants, $a, b$, and $c$, are to be calculated from measured values of $E$ at the freezing point of antimony and at the silver and gold points. The antimony used in determining these constants shall be such that its freezing temperature, determined with a standard resistance thermometer, is not lower than $630.3^{\circ} \mathrm{C}$. Alternatively the thermocouple may be calibrated by direct comparison with a standard resistance thermometer in a bath at any uniform temperature between $630.3^{\circ}$ and $630.7^{\circ} \mathrm{C}$.

The platinum wire of the standard thermocouple shall be annealed and of such purity that the ratio $R_{100} / R_{0}$ is greater than 1.3910 . The alloy wire shall consist nominally of 90 percent platinum and 10 percent rhodium by weight. When one junction is at $0^{\circ} \mathrm{C}$, and the other at the freezing point of antimony $\left(630.5^{\circ} \mathrm{C}\right)$, silver, or gold, the completed thermocouple shall have electromotive forces, in microvolts, such that

$$
\begin{gathered}
E_{\mathrm{Au}}=10,300 \pm 50 \mu v \\
E_{\mathrm{Au}}-E_{\mathrm{Ag}}=1185+0.158\left(E_{\mathrm{Au}}-10,310\right) \pm 3 \mu v \\
E_{\mathrm{Au}}-E_{\mathrm{Sb}}=4776+0.631\left(E_{\mathrm{Au}}-10,310\right) \pm 5 \mu v
\end{gathered}
$$


(d) Above the gold point the temperature, $t$, is defined by the formula

$$
\frac{J_{t}}{J_{\mathrm{Au}}}=\frac{e^{\frac{c_{2}}{\lambda\left(t_{\mathrm{Au}}+T_{0}\right)}}-1}{e^{\frac{c_{2}}{\lambda\left(t+T_{0}\right)}}-1},
$$

in which

$J_{t}$ and $J_{\mathrm{Au}}$ are the radiant energies per unit wavelength interval at wavelength, $\lambda$, emitted per unit time by unit area of a black body at the temperature, $t$, and at the gold point, $t_{\mathrm{Au}}$, respectively.

$c_{2}$ is $1.438 \mathrm{~cm}$ degrees.

$T_{0}$ is the temperature of the ice point in ${ }^{\circ} \mathrm{K}$. $\lambda$ is a wavelength of the visible spectrum.

$e$ is the base of Naperian logarithms.

\section{Part III. Recommendations}

The recommendations in the following sections are advisory rather than mandatory. The apparatus, methods, and procedures recommended represent good practice at this time, but there is no intention of retarding the development and use of improvements and refinements. Experience has shown these recommendations to be in the interest of uniformity and reproducibility in the realization of the temperature scale defined in part II.

\section{Standard Resistance Thermometer}

A standard resistance thermometer should be so designed and constructed that the wire of the platinum resistor is as nearly strain-free as practicable and will remain so during continued use. The most suitable platinum wire is that drawn from a fused ingot, not from forged sponge.

Satisfactory thermometers have been made with wire as small as $0.05 \mathrm{~mm}$ and as large as $0.5 \mathrm{~mm}$ in diameter, a short portion of each lead adjacent to the resistor being made of platinum and continuing with gold wire through the region of temperature gradient. The completed resistor of the thermometer should be annealed in air at a temperature not lower than about $450^{\circ} \mathrm{C}$, or if it is to be used at temperatures above $450^{\circ} \mathrm{C}$, at a temperature higher than the highest temperature at which it is to be used. It is recommended that the tube protecting the completed resistor be filled with gas containing some oxygen.

A useful criterion, which serves as a safeguard against inferior construction of the completed thermometer and against errors in the calibrations at the fixed points, is that $\left(R_{\mathrm{S}}-R_{0}\right) /\left(R_{100}-R_{0}\right)$ (where $R_{\mathrm{S}}$ is the resistance at the sulfur point) should be between 4.2165 and 4.2180. Similarly, if the thermometer is calibrated for use in the range below $0^{\circ} \mathrm{C}$, the ratio, $\left(R_{\mathrm{s}}-R_{\mathrm{O}_{2}}\right) /\left(R_{100}-R_{0}\right)$ (where $R_{\mathrm{O}_{2}}$ is the resistance at the oxygen point) should be between 6.143 and 6.144. The constancy of resistance at a reference point, such as the triple point of water (or the ice point), before and after use at other temperatures, is also a valuable criterion of the adequacy of the annealing and the reliability of the thermometer in service.

\section{Standard Thermocouple}

Satisfactory standard thermocouples have been made of wires not less than $0.35 \mathrm{~mm}$ and not more than $0.65 \mathrm{~mm}$ in diameter. Before calibration, the wires of the couple should be annealed in air for an hour at about $1100^{\circ} \mathrm{C}$. The wire of the thermocouple should be mounted so as to avoid all mechanical constraints in the region where steep temperature gradients are likely to occur.

\section{Pressure}

Each of the fixed points is given as a temperature of equilibrium at a pressure of 1,013,250 dynes/ $\mathrm{cm}^{2}$. This pressure corresponds to the pressure exerted by a column of mercury $760 \mathrm{~mm}$ high, having a density of $13.5951 \mathrm{~g} / \mathrm{cm}^{3}$ and subject to a gravitational attraction of 980.665 dynes/g. Except for work of the highest precision, pure ordinary (commercial) mercury may be taken as having a mean density of $13.5951 \mathrm{~g} / \mathrm{cm}^{3}$ at $0^{\circ} \mathrm{C}$ in such a mercury column.

In the following sections concerning fixed points, the formulas giving the relation between the pressure, $p_{t}$, at the midpoint of the platinum resistor and the corresponding equilibrium temperature, $t_{p}$, are given in two forms. The poly nomial is a convenient form when the pressure is near 1 standard atmosphere, whereas the logarithmic form is applicable, as is well known, over a much larger range. Both of these forms are given as functions of the ratio of $p$ to $p_{0}$ (standard atmospheric pressure) and, consequently, are equally applicable whether $p$ and $p_{0}$ are expressed in dynes $/ \mathrm{cm}^{2}$ or in $\mathrm{mm}$ of mercury at $0^{\circ} \mathrm{C}$ and under a gravitational attraction of 980.665 dynes/g. 


\section{Zero Point of the Scale}

The temperature $0^{\circ} \mathrm{C}$ may be realized experimentally well enough for nearly all purposes by the use of a mixture of finely divided ice and water saturated with air at $0^{\circ} \mathrm{C}$ in a well-insulated container such as a Dewar flask. It is recommended, however, that for work of the highest precision the zero point be realized by means of the triple point of water, a point to which the temperature $+0.0100^{\circ} \mathrm{C}$ has been assigned.

This value agrees with all existing experimental determinations up to the present time within about 0.0002 degree.

\section{(a) Triple Point of Water}

The temperature of equilibrium between ice, liquid water, and water vapor has been realized in glass cells from 4 to $7 \mathrm{~cm}$ in diameter, which have an axial reentrant well for thermometers and contain only water of high purity. The amount of water should be such as to permit adequate immersion of the thermometer and to ensure the existence of the three phases during measurements. Such cells, when properly prepared for use and kept entirely immersed in an ordinary ice bath, have been found to be capable of maintaining a temperature constant to 0.0001 degree for several days.

Cells have been prepared for use by cooling the entire contents until small crystals are present throughout the liquid. A preferred method is to freeze a thick mantle of ice around the well by rapid cooling from within. With this method, the water ahead of the forming ice contains most of the impurities initially in the water frozen. This will result in an appreciable lowering of the temperature at the outside of the mantle unless the initial purity of the water is sufficiently high. If a thin layer of the pure ice next to the well is then melted, a very pure water-ice interface immediately surrounding the well is obtained, and this fixes a temperature that is constant and reproducible to better than 0.0001 degree, provided the initial outside temperature is not more than 0.001 degree lower than the temperature produced on the interior after melting the thin layer of ice.

The equilibrium temperature, $t$, corresponding to the depth, $H$ (in $\mathrm{mm}$ ), below the vapor-liquid surface, may be calculated from the formula

$$
t=0.0100-0.7 \times 10^{-6} H .
$$

(b) Ice Point

By observing precautions in regard to purity of ice and water, the saturation of the water at $0^{\circ} \mathrm{C}$ with uncontaminated air, and the effect of pressure, a temperature reproducible within a few tenthousandths of a degree may be realized.

The effect of pressure may be calculated from the formula ${ }^{3}$

$$
t=0.0099\left(1-\frac{p}{p_{0}}\right)-0.7 \times 10^{-6} H,
$$

where $t$ is the equilibrium temperature, $p$ is the prevailing atmospheric pressure at which the water is saturated with air, and $H$ is the depth (in $\mathrm{mm}$ ) below the surface of the mixture of water and ice.

\section{Oxygen Point}

The temperature of equilibrium between liquid oxygen and its vapor is usually realized experimentally by the static method. The platinum resistor of the standard thermometer and the free surface of the liquid oxygen in its container are brought to the same temperature in a suitable cryostat, such as a metal block in a well-stirred bath of liquid oxygen. The tube which connects the space containing the pure liquid oxygen to the manometer used for the measurement of the vapor pressure should be protected against temperatures lower than the temperature of the pure liquid oxygen at the location of the resistor of the standard resistance thermometer.

The equilibrium temperature, $t_{p}$, corresponding to a pressure, $p$, may be found to an accuracy of a few thousandths of a degree over the range from $p=660$ to $p=860 \mathrm{~mm}$, by means of the formula

$$
\begin{aligned}
& t_{p}=-182.970+9.530\left(\frac{p}{p_{0}}-1\right)-3.72\left(\frac{p}{p_{0}}-1\right)^{2} \\
& +2.2\left(\frac{p}{p_{0}}-1\right)^{3},
\end{aligned}
$$

or to the same accuracy but over a wider range by the formula

$$
t_{p}=-182.970+\frac{21.94 \log _{10} \frac{p}{p_{0}}}{1-0.261 \log _{10} \frac{p}{p_{0}}} .
$$

\footnotetext{
${ }^{3}$ Author's note. In this formula the value, 0.0099 , which appears in the official text, should be 0.010 . Ordinarily the incorrect value will not lead to a significant error.
} 


\section{Steam Point}

The temperature of equilibrium between liquid water and its vapor has been realized by the dynamic method with the thermometer in the saturated vapor, using apparatus of various designs, some closed and others open to the atmosphere. Closed systems, in which a steam-point apparatus and manometer are connected to a manostat of large volume filled with helium are preferable for precise calibrations at the steam point.

The design of the steam-point apparatus should be such as to avoid any superheating of the water vapor around the thermometer, contamination with air or other impurities, and radiation effects. A criterion that the equilibrium temperature has been attained is that the observed temperature corrected to a constant pressure is independent of elapsed time, of variations in the heat supply to the liquid, of variations in the heat loss from the walls, and of the depth of immersion of the thermometer.

The equilibrium temperature, $t_{p}$, corresponding to the pressure, $p$, may be found to an accuracy within 0.001 degree over the range from $p=660$ to $p=860 \mathrm{~mm}$ by means of the formula

$$
\begin{aligned}
t_{p}= & 100+28.012\left(\frac{p}{p_{0}}-1\right)-11.64\left(\frac{p}{p_{0}}-1\right)^{2} \\
& +7.1\left(\frac{p}{p_{0}}-1\right)^{3},
\end{aligned}
$$

or to the same accuracy over a wider range by the formula

$$
t_{p}=100+\frac{64.500 \log _{10} \frac{p}{p_{0}}}{1-0.1979 \log _{10} \frac{p}{p_{0}}} .
$$

\section{Sulfur Point}

The sulfur used in a sulfur boiling-point apparatus should not contain more than 0.005 percent of impurities. Selenium and arsenic are the impurities that have been found most likely to be present in quantities sufficient to affect the temperature of equilibrium to a significant extent.

In the conventional type of sulfur boilingpoint apparatus which has proved satisfactory for an accuracy of 0.01 to 0.02 degree, the sulfur is contained in a tube of glass, fused silica, or similar material with an internal diameter of 4 to $5 \mathrm{~cm}$.
The length of the tube is determined by the consideration that the vapor column must be long enough to accommodate a radiation shield and to permit the necessary depth of immersion of the resistance thermometer. Electric heating is preferable. Above the source of heat the tube is surrounded by heat-insulating material.

In a recent work in which an accuracy of about 0.001 degree was desired, the thermometer was not immersed directly in the sulfur vapor, but in an aluminum thermometer well, thus adapting the apparatus for use with a closed system. The thermometer well was provided with one or more radiation shields so designed that the interior of the shield approximated a black body, but with ample openings for circulation of sulfur vapor throughout the interior. An electric heater was provided to control the heat loss from the walls.

A criterion that equilibrium temperatures have been attained is that the observed temperatures corrected to a constant pressure should be independent of elapsed time, of variations in the heat supply to the liquid, of variations in the heat loss from the walls, and of the depth of immersion of the thermometer.

The equilibrium temperature, $t_{p}$, corresponding to a pressure, $p$, may be found to an accuracy of about 0.001 degree over the range from $p=660$ to $p=800 \mathrm{~mm}$ by means of the formula

$$
\begin{aligned}
t_{p}= & 444.6+69.010\left(\frac{p}{p_{0}}-1\right)-27.48\left(\frac{p}{p_{0}}-1\right)^{2}+ \\
& 19.14\left(\frac{p}{p_{0}}-1\right)^{3},
\end{aligned}
$$

or, over a wider range, by the formula

$$
t_{p}=444.6+\frac{158.92 \log _{10} \frac{p}{p_{0}}}{1-0.234 \log _{10} \frac{p}{p_{0}}} .
$$

\section{Silver and Gold Points}

Data on the effect of the impurities most likely to be present in carefully purified silver or gold indicate that the addition of 0.01 percent by weight of metallic impurities to pure silver, or of 0.005 percent to pure gold will probably not change the freezing point in either case by more than 0.1 degree.

For calibrating a thermocouple, the metal is contained in a crucible of pure graphite or other 
refractory material which will not contaminate the metal. Crucibles of artifical graphite about $3 \mathrm{~cm}$ inside diameter and $15 \mathrm{~cm}$ deep with a wall about $1 \mathrm{~cm}$ thick have been found very satisfactory. The amount of metal required in such a crucible is about $1600 \mathrm{~g}$ of gold or about $900 \mathrm{~g}$ of silver. Silver must be protected from oxygen while hot.

The crucible and metal are placed in a furnace capable of heating the contents to a uniform temperature. The metal is melted and brought to a uniform temperature a few degrees above its melting point, then allowed to cool slowly. The thermocouple, mounted in a porcelain tube with porcelain insulators separating the two wires, is immersed in the molten metal through a hole in the center of the crucible cover. The depth of immersion should be such that the observed electromotive force of the thermocouple is not changed by more than 1 microvolt when the immersion is increased or decreased by $1 \mathrm{~cm}$. During freezing, the electromotive force should remain constant within 1 microvolt for a period of at least 5 minutes.

\section{Freezing Point of Antimony}

The procedure in using the freezing point of antimony as a calibration temperature is substantially the same as outlinéd above for the silver and gold points. Antimony has a marked tendency to undercool before freezing. The undercooling will not be excessive if the metal is heated only a few degrees above its melting point and if the liquid metal is stirred. During freezing, the electromotive force should remain constant within 1 microvolt for a period of at least 5 minutes.

\section{Temperature of the Ice Point on the Kelvin Scale}

For the sake of uniformity, it is recommended that the temperature of the ice point on the Kelvin scale be taken as $273.15^{\circ} \mathrm{K} .{ }^{4}{ }^{5}$

\section{Part IV. Supplementary Information}

\section{Resistance-Temperature Formulas}

The interpolation formula, for the range $0^{\circ} \mathrm{C}$ to the freezing point of antimony, as given in the definition of the scale,

$$
R_{t}=R_{0}\left(1+A t+B t^{2}\right),
$$

may be written in the Callendar form

$$
t=\frac{1}{\alpha}\left(\frac{R_{t}}{R_{0}}-1\right)+\delta\left(\frac{t}{100}-1\right)\left(\frac{t}{100}\right),
$$

where

$$
\alpha=\frac{1}{100}\left(\frac{R_{100}}{R_{0}}-1\right) .
$$

The relations between the coefficients are

$$
\begin{array}{ll}
A=\alpha\left(1+\frac{\delta}{100}\right), & \alpha=A+100 B, \\
B=-\frac{\alpha \delta}{100^{2}}, & \delta=-\frac{100^{2} B}{A+100 B} .
\end{array}
$$

The condition that $R_{100} / R_{0}$ shall be greater than 1.3910 is equivalent to requiring that $\alpha$ shall be greater than 0.003910 . The condition that $\left(R_{\mathrm{s}}-R_{0}\right) /\left(R_{100}-R_{0}\right)$ shall be between 4.2165 and 4.2180 is equivalent to requiring that $\delta$ shall be between 1.488 and 1.498 .

Similarly, the interpolation formula, for the range $0^{\circ} \mathrm{C}$ to the oxygen point, as given in the definition of the scale,

$$
R_{t}=R_{0}\left[1+A t+B t^{2}+C(t-100) t^{3}\right]
$$

may be written in the Callendar-Van Dusen form

$$
\begin{aligned}
t= & \frac{1}{\alpha}\left(\begin{array}{l}
R_{t} \\
R_{0}
\end{array}-1\right)+\delta\left(\frac{t}{100}-1\right) \frac{t}{100}+ \\
& \beta\left(\frac{t}{100}-1\right)\left(\frac{t}{100}\right)^{3} .
\end{aligned}
$$

\footnotetext{
1 This value $273.15^{\circ} \mathrm{K}$ was not ratified by either the International Committee or the General Conference. The choice of the last decimal figure was postponed.

${ }^{5}$ Author's note. The value $273.15^{\circ} \mathrm{K} \pm .02$ for the temperature of the ice pcint was originally proposed by the Advisory Committee on Thermometry in 1939; and, in 1948, the Committee did not consider that encugh new knowledge had been gained to change this value. That some uncertainty was felt about this action is indicated by a clause in Resolution 2, which already had been approved by the Advisory Committee, and was later adopted by the General Conference. This resolution resulted from a proposal the committee had received to define an absolute thermodynamic temperature scale by assigning a value to the temperature of a single fixed point.

"Resolution 2. The Advisory Committee recognizes the principle of an absolute thermodynamic scale requiring only one fundamental fixed point, which would now be the triple point of pure water, for which the absolute temperature will be chosen later.

"The intreduction of this new scale in no way affects the International Scale which continues to be the recommended practical scale."

This resolution defers the establishment of a precise value for the temperature cf the ice point. Inasmuch as it is definitely stated at the beginning of Part III of this text that "The recommendations in the following sections are advisory rather than mandatory", many scientists will not consider it mandatory to accept this recommended value for the ice point. In the United States of America the value, $273.16^{\circ} \mathrm{K}$, for the temperature of the ice point has been so generally accepted that its use now prevails in scientific publications, and this value will probably continue to be used until a more accurate one has been agreed upon.
} 
The relations between $A, B$ and $\alpha, \delta$ are as given above and the other relations are

$$
C=-\frac{\alpha \beta}{100^{4}}, \text { and } \beta=-\frac{100^{4} C}{A+100 B}
$$

The condition that $\left(R_{\mathrm{S}}-R_{\mathrm{O}_{2}}\right) /\left(R_{100}-R_{0}\right)$ shall be between 6.143 and 6.144 is equivalent to requiring that $0.5852 \delta-\beta$ shall be between 0.7656 and 0.7598 .

\section{Secondary Fixed Points}

In addition to the six fundamental and primary fixed points, certain other fixed points are available and may be useful for various purposes. Some of the more constant and reproducible of these fixed points and their temperatures on the International Temperature Scale of 1948 are given in Table II. The temperatures given are those corresponding to a pressure of 1 standard atmosphere, except for the triple points of water and of benzoic acid. The formulas for the variation of temperature with pressure are intended for use over the range from $p=680$ to $p=780 \mathrm{~mm}$ of mercury.

Table II. Secondary Fixed Points under the pressure of 1 standard atmosphere (except for the triple points)

Temperature ${ }^{\circ} \mathrm{C}$

(Int. 1948)

Temperature of equilibrium between solid carbon dioxide and its vapor

$$
t_{p}=-78.5+12.12\left(\frac{p}{p_{0}}-1\right)-6.4\left(\frac{p}{p_{0}}-1\right)^{2}
$$

Temperature of freezing mercury _. _. _ _ _ _ _ -38.87

Temperature of equilibrium between ice, water and its vapor (Triple Point) ............

Temperature of transition of sodium sulphate decahydrate.

Temperature of triple point of benzoic acid___ $\quad 122.36$

Temperature of equilibrium between naphthalene and its vapor. 218. 0

$$
t_{D}=218.0+44.4\left(\frac{p}{p_{0}}-1\right)-19\left(\frac{p}{p_{0}}-1\right)^{2}
$$

Temperature of freezing tin _............

Temperature of equilibrium between benzophenone and its vapor

$$
t_{p}=305.9+48.8\left(\frac{p}{p_{0}}-1\right)-21\left(\frac{p}{p_{0}}-1\right)^{2}
$$

Temperature of freezing cadmium 320. 9
Table II. Secondary Fixed Points under the pressure of 1 standard atmosphere-Continued

Temperature ${ }^{\circ} \mathrm{C}$

(Int. 1948)

Temperature of freezing lead. 327. 3

Temperature of equilibrium between mercury and its vapor.

356. 58

$$
\begin{aligned}
t_{p}= & 356.58+55.552\left(\frac{p}{p_{0}}-1\right)- \\
& 23.03\left(\frac{p}{p_{0}}-1\right)^{2}+14.0\left(\frac{p}{p_{0}}-1\right)^{3}
\end{aligned}
$$

Temperature of freezing zinc

Temperature of freezing antimony

419. 5

Temperature of freezing aluminum 630. 5

Temperature of freezing copper in a reducing atmosphere _._.

Temperature of freezing nickel_ . . _ _._. _... 1453

Temperature of freezing cobalt _..._. _....... 1492

Temperature of freezing palladium_______ 1552

Temperature of freezing platinum _ _ _ _ _ _ 1769

Temperature of freezing rhodium _..._. _ _ 1960

Temperature of freezing iridium _......... 2443

Temperature of melting tungsten_.......... 3380

3. Relation Between the International Temperature Scale of 1948 and the Thermodynamic Centigrade Scale

At the time of the adoption of the International Temperature Scale of 1927, the evidence available was insufficient to establish definite differences between that scale and the thermodynamic centigrade scale. The earlier investigations, such as those published in 1911 by the PhysikalischTechnische Reichsanstalt, indicated no differences exceeding 0.05 degree in the range $0^{\circ} \mathrm{C}$ to the sulfur point.

Recent investigations at the Massachusetts Institute of Technology indicate larger differences between $200^{\circ} \mathrm{C}$ and the sulfur point. Intercomparisons of two nitrogen gas thermometers with platinum resistance thermometers were made at $0^{\circ}, 25^{\circ}, 50^{\circ}, 75^{\circ}, 100^{\circ}, 150^{\circ}, 200^{\circ}, 250^{\circ}, 300^{\circ}$, $350^{\circ} \mathrm{C}$, the mercury boiling point, $400^{\circ} \mathrm{C}$ and the sulfur point. The differences found between $t$ (thermodynamic scale) and $t$ (International Scale) have been formulated as follows:

$$
\begin{aligned}
t(\text { therm })-t(\operatorname{In} \mathrm{t})= & \frac{t}{100}\left(\frac{t}{100}-1\right)(0.04217- \\
& \left.7.481 \times 10^{-5} t\right) .
\end{aligned}
$$

The sulfur point on the thermodynamic scale was found to be $444.74^{\circ} \mathrm{C}$, the results obtained with the two gas thermometers differing by about 0.05 degree. 
In the range from $0^{\circ} \mathrm{C}$ to the oxygen point, intercomparisons published by the Physikalisch-Technische Reichsanstalt in 1932 and by the Leiden Laboratory in 1935 indicate that the differences between the International and thermodynamic scales are less than 0.05 degree. Agreement is lacking as to the sign of some of the reported differences, which are of the order of magnitude of the possible uncertainties in the gas thermometer measurements.

In the range extending below the oxygen point, there is evidence that temperatures on the International Temperature Scale of 1927 are progressively higher than those on the thermodynamic scale, amounting to several hundredths of a degree at $-190^{\circ} \mathrm{C}$. For this reason, and also because it is advantageous to terminate the various parts of the scale at calibration points, the International Temperature Scale of 1948 extends only to the oxygen point.

In the range from the freezing point of antimony to the gold point, there is little evidence relative to the sign or magnitude of departures of the International from the thermodynamic scale. The value $1063.0^{\circ} \mathrm{C}$ for the gold point has been accepted as a conventional definition and will doubtless remain so until the appearance of new and more accurate basic data. The change from $960.5^{\circ}$ to $960.8^{\circ} \mathrm{C}$ is well within the uncertainty of the location of the silver point on the thermodynamic scale. This change makes the thermocouple scale join more smoothly, not only with the resistance thermometer scale at the freezing point of antimony, but also with the optical pyrometer scale at the gold point when $c_{2}=1.438 \mathrm{~cm}$ degrees.

It is of interest to note that, granting the validity of Planck's formula, temperatures above the gold point on the International Temperature Scale of 1948 will differ from those on the thermodynamic centigrade scale only to the extent caused by errors in the constants, $c_{2}, t_{\mathrm{Au}}$, and $T_{0}$, used in the formula.

Washington, January 14, 1949. 